\title{
Improvement of reliability in pressure measurements and international mutual recognition
}

\author{
— Incorporation of industrial digital pressure gauges \\ to the national metrology system -
}

\author{
Tokihiko KoBATA*, Momoko KoJIMA and Hiroaki KAJIKAWA
}

[Translation from Synthesiology, Vol.4, No.4, p.209-221(2011)]

\begin{abstract}
Reliable pressure measurements provide the basis for all activities in society and industry, and every country shows considerable interest in them in international trade. Recently, characteristics of industrial digital pressure gauges and the short- and long-term stabilities as well as the stability under environment change and during transportation have been improved remarkably. AIST focused its attention on the technological advancement, and, after evaluating the characteristics of digital pressure gauges in detail, incorporated them to some phases in the metrology system, which are the upgrading of national pressure standards, the international comparisons of the standards and the domestic traceability system. Consequently, the reliability of pressure measurements in industrial fields has been secured through an efficient process, and the international mutual recognition has been promoted by carrying out many international comparisons.
\end{abstract}

Keywords : Pressure standard, digital pressure gauge, calibration, international comparison, metrological traceability

\section{Introduction}

The measurement of pressure is one of the essential measurements in people's lives, society, industry, and science and technology. The pressure measurements for safety and security include the atmospheric measurement in meteorological observation, the altitude measurement for aircrafts, the blood pressure in medicine and health management, the measurement for high-pressure gas control, and the pressure measurement in the manufacturing process of food and pharmaceutical products. The pressure measurements for energy savings include the air conditioning of buildings and the adjustment of air pressure in tires and automobile engines. Moreover, various pressure measurements are conducted at various sites such as the maintenance of clean rooms where high air purity must be maintained for the manufacture of semiconductors and precision devices, the measurement and control of pressure in various processing, and the inspection of plants. In the field of science and technology, various R\&Ds using pressure control and measurements are being done.

For the pressure measurement conducted at the industrial sites, diverse pressure gauges are employed for wide pressure ranges, from low pressure of $1 \mathrm{~Pa}$ or less to high pressure of $1 \mathrm{GPa}$ or more. Recently, the performance of the industrial pressure gauge increased significantly. Specifically, the performance increased in terms of expansion of measurement range, improvement in high resolution, improved short- and long-term stability, and improved stability in environment change and during transportation.
AIST has focused on the improvements of the performance of such digital pressure gauge, and has spent effort on the precise evaluation of its properties. As a result, the digital pressure gauge is now utilized in the upgrading of national standard and the standard provision, and the reliability of pressure measurement at the industrial sites improved greatly. On the other hand, the international mutual recognition of the measurement results is essential in modern international trade. AIST has contributed greatly in providing technical data for the mutual recognition of the national standard, such as conducting various international comparisons using the digital pressure gauge.

In this paper, the R\&D for the digital pressure gauge and its results in improving the reliability of pressure measurement at the industrial sites and the efforts on international mutual recognition to ensure free trade will be presented from the perspective of synthesiology based on the scenario.

\section{Demand for the reliability of pressure measurement}

\subsection{Demand from the Japanese industry}

To conduct highly reliable pressure measurement using the pressure gauge at the industrial site, it is necessary to correctly calibrate the pressure gauge so it will be traceable to the national standard for pressure owned by AIST. On the other hand, the main standard for pressure owned by industry is the highly precise device called the pressure balance

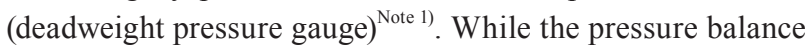

National Metrology Institute of Japan, AIST Tsukuba Central 3, 1-1-1 Umezono, Tsukuba 305-8563, Japan

* E-mail : tokihikokobata@aist.go.jp

Original manuscript received July 4, 2011, Revisions received October 12, 2011, Accepted October 14, 2011 
is characterized by its excellent long-term stability, the device itself is heavy and large, and for the client receiving the calibration service, transporting this bulky device to the calibration lab is troublesome. Also, the calibration at the calibration lab may take about two months at the longest, and since the standard device cannot be used during that time, extra standard device must be kept for backup, requiring significant amount of expenditure. Therefore, industry demanded some method where calibration could be done effectively at the same precision as the conventional method, without transporting the pressure balance.

Recently, the property of the industrial digital pressure gauge improved significantly, and there is good prospect of maintaining stability during transportation and in environment change, as well as certain degree of long-term stability. Therefore, we attempted to incorporate and utilize the industrial digital pressure gauge into the standard system. Normally, the digital pressure gauge is transported to the user's site after being calibrated using the standard device at the calibration lab. Therefore, if the displayed value of the digital pressure gauge after being transported is sufficiently stable, the user will be able to maintain the calibration value that is traceable to the national standard at the same precision as before, without having to transport the heavy and large pressure balance to the calibration lab. This will ensure high efficiency of the calibration work.

To promote the use of digital pressure gauge in the standard system, the necessary conditions are: the stability of the calibration value is sufficiently maintained even when the industrial digital pressure gauge is transported anywhere in Japan, and sufficient stability is maintained until the next calibration is conducted. AIST embarked on the research to determine whether the industrial digital pressure gauge had such properties.

\subsection{International demand}

With the globalization of economy, production, and trade, there is rising interest in the conformity of the measurement results by various measuring equipment among different

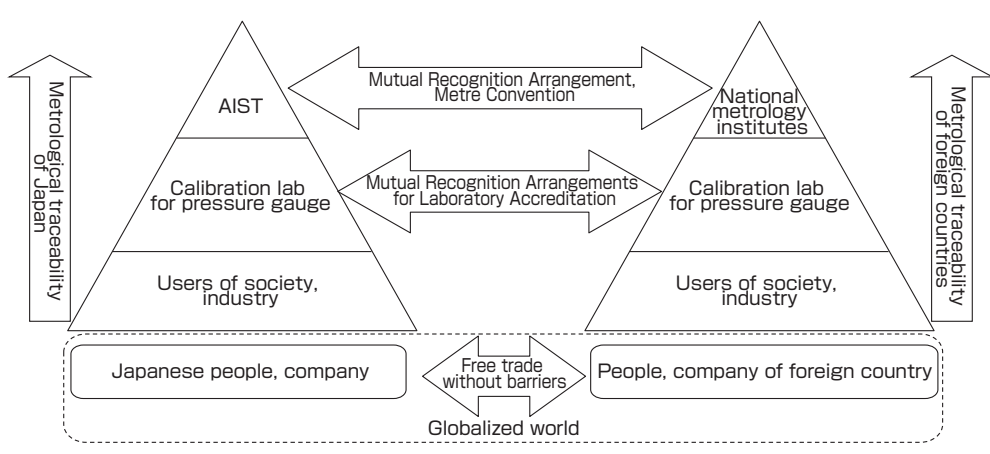

Fig. 1 Framework of the international mutual recognition for metrological standard countries $^{[1][2]}$. For example, at Narita and Haneda Airports, there is an increase in international business such as the maintenance of the aircrafts of foreign companies by the maintenance shops of the Japanese airline companies. Several aircraft accidents occurred in the US in the 1990s, and the US government revised the Federal Aviation Law to maintain the safety of the US citizens, and the requirements for maintaining the US-owned aircrafts demanded that the measuring devices such as the pressure gauges to be traceable to the national standard of the National Institute of Standards and Technology (NIST), the American national metrology institute. The inspectors of the Federal Aviation Administration (FAA) would conduct regular on-site inspection of the maintenance shops. The demand of the US government was applied not only to the American airline companies but also to the foreign maintenance companies that maintained the US-owned aircrafts. Japan Airlines and All Nippon Airways had to make almost all the measuring devices in their maintenance shops traceable to NIST. This became an international issue, since most of the measuring devices used at the maintenance shops of the Japanese airline companies were made in Japan, and were traceable to AIST (at that time, the laboratory for former Agency of Industrial Science and Technology). The Japanese government stated that the Japanese national standard was proven to be equivalent to the American standard, and the devices traceable to the Japanese national standard should be allowed. This claim was accepted as an individual case.

This issue spread to the airline companies around the world. With the background of such an issue, the Mutual Recognition Arrangement (MRA) of the Comité International des Poids et Mesures (CIPM) under the Convention du Mètre was established, to mutually recognize the equivalence of the national standards owned by the national metrology institutes around the world ${ }^{[3]}$. Figure 1 shows the framework of the international mutual recognition of the metrological standards.

In this framework, the technological requirement for mutual recognition was the actual comparison of the national standards among the countries (international comparison). The results of the international comparisons and the calibration and measurement capabilities recognized for participating institutes are registered in the Appendix B and $\mathrm{C}$ of the international MRA. This can be viewed on the Key Comparison Database (http://kcdb.bipm.fr/) of the Bureau International des Poids et Mesures (BIPM). The level of the national standard owned by each country can be known from this technological information.

As the Japanese metrology institute, AIST aims to establish and provide the national standard for pressure and to organize the traceability system of pressure measurement in Japan. By participating actively in the international 
comparisons, the international equivalence of the Japanese national standard for pressure has been demonstrated in the aforementioned Key Comparison Database. AIST is also responsible for maintaining the national interest by displaying the capabilities in calibration and measurement that are recognized by other countries.

With this background, we have been involved in advancing the national standards and in demonstrating the equivalence of the national standards with other countries, and we decided to utilize the industrial digital pressure gauge that has shown significant increase in performance.

\subsection{Technological issues}

The national standard for pressure ${ }^{[4][5]}$ realized by AIST is for stationary fluid where hydrostatic pressure mediated by gas or liquid is established. In general, gas is used as the medium of pressure standard for low pressure, and liquid for high pressure. AIST also establishes the vacuum standard for pressure with small absolute pressure ${ }^{[6]}$.

At the time of the establishment of AIST in 2001, the national standard was established for the pressure range from $5 \mathrm{kPa}$ to $500 \mathrm{MPa}$ using multiple pressure standard devices, and the calibration service was provided. With the advancement of science and industrial technology, various improvements such as the increased types of pressure standards, the expansion of pressure range, and the decrease of uncertainty were requested by the users at the industrial sites, according to the survey of demand for physical standards conducted in $2002^{[7]}$. Specifically, there were many requests for low-pressure standard and differential pressure standard for $10 \mathrm{kPa}$ or less that were necessary for air conditioning, pharmaceuticals, medicine, and semiconductors. There were also requests for high-pressure standard surpassing $500 \mathrm{MPa}$ for the development of internal combustion engines, as well as in the fields of materials, manufacturing, and machine processing.

AIST upgraded the national standards by conducting new technological development ${ }^{[8]-[10]}$. Currently, the pressure range of $1 \mathrm{~Pa}$ or $1 / 100,000$ of atmospheric pressure to $1 \mathrm{GPa}$ or about 10,000 times the atmospheric pressure are covered, using various pressure standard devices. To check the conformity of the generated pressure of these national standard devices,

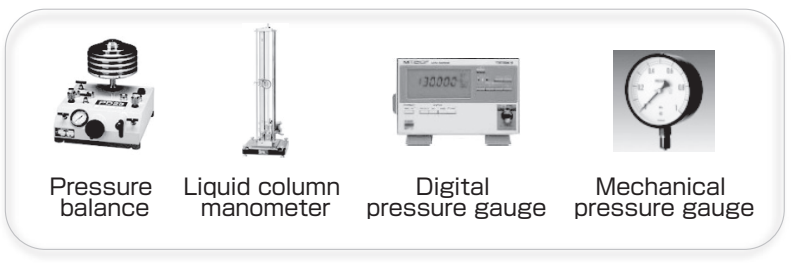

Fig. 2 Examples of various pressure gauges the comparative measurement among the standard devices is necessary, and efficiency and advancement were issues in managing the multiple standard devices.

At the field measurement of pressure, calibration and provision of standards that were effective yet with little burden on the user as much as possible were desired due to the increased number of devices that must be calibrated. As mentioned earlier, there have been recent new technological developments by companies in Japan and overseas, and the reliability of industrial digital pressure gauge using various pressure sensors increased. The high-precision pressure gauge with high resolution of six digits or more against full scale and with excellent long-term stability is now available. Therefore, AIST experimentally investigated whether such high-precision industrial digital pressure gauges could be used to increase the efficiency and performance of pressure standard maintenance, and whether it satisfied the requirement as standard devices.

\section{Various pressure gauges}

Figure 2 shows the various pressure gauges that are used at the site of pressure measurement.

The measuring device that is independently capable of conducting the absolute measurement of physical quantity without calibration by other measuring device is called the primary measuring device. Various devices are considered as primary measuring device for pressure (or primary pressure gauge), and the main ones are pressure balance ${ }^{[1]]}$ and liquid column manometer. Figure 3 illustrates the principles of the pressure balance and the liquid column manometer. On the other hand, the measuring device that is not independently capable of conducting the absolute measurement of physical quantity and requires calibration by primary measuring device is called the secondary measuring device. The secondary measuring device for pressure (secondary pressure gauge) includes digital pressure gauge ${ }^{[12]}$ and mechanical pressure gauge (Bourdon tube pressure gauge ${ }^{[13]}$ ).

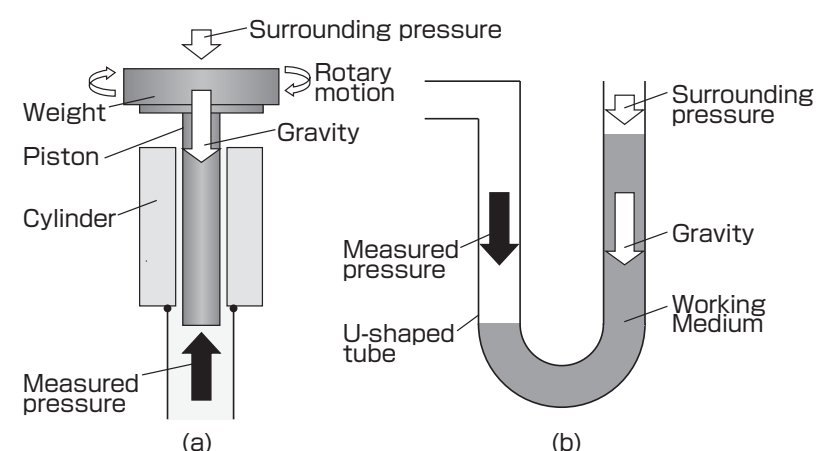

(a)

(b)

Fig. 3 Principles of pressure balance (a) and liquid column manometer (b) 
Table 1. Characteristics of various pressure gauges

\begin{tabular}{l|llll}
\hline $\begin{array}{l}\text { Types of } \\
\text { pressure } \\
\text { gauges }\end{array}$ & $\begin{array}{l}\text { Types of } \\
\text { measuring } \\
\text { instrument }\end{array}$ & $\begin{array}{l}\text { Maximum } \\
\text { pressure }\end{array}$ & $\begin{array}{l}\text { Size,weight, } \\
\text { operability }\end{array}$ & $\begin{array}{l}\text { Automation, } \\
\text { systemization }\end{array}$ \\
\hline $\begin{array}{l}\text { Pressure } \\
\text { balance }\end{array}$ & Primary & Over $1 \mathrm{GPa}$ & $\begin{array}{l}\text { Large, heavy, } \\
\text { difficult }\end{array}$ & Difficult \\
\hline $\begin{array}{l}\text { Liquid column } \\
\text { manometer }\end{array}$ & Primary & $300 \mathrm{kPa}$ & $\begin{array}{l}\text { Large, heavy, } \\
\text { difficult }\end{array}$ & Difficult \\
\hline $\begin{array}{l}\text { Digital pressure } \\
\text { gauge }\end{array}$ & Secondary & Over $1 \mathrm{GPa}$ & $\begin{array}{l}\text { Small, light, } \\
\text { easy }\end{array}$ & Easy \\
\hline $\begin{array}{l}\text { Mechanical } \\
\text { pressure gauge }\end{array}$ & Secondary & Over $1 \mathrm{GPa}$ & $\begin{array}{l}\text { Small, light, } \\
\text { easy }\end{array}$ & Difficult \\
\hline
\end{tabular}

The pressure balance is a measuring device that is capable of highly precise generation of pressure. The reliability of measurement is high, and it is used as the national standard for pressure in many countries. It is also used widely as the standard device at the industrial sites. The basic components of the pressure balance are piston, cylinder, and weights with known mass. The exterior surface of the piston and the interior surface of the cylinder are precisely machined to maintain good circularity and cylindricity. Normally, when generating pressure with the pressure balance, the piston and the weights are rotated after floating the piston, which is under downward gravity by the piston and weights, to appropriate position by upward pressure. By doing so, the mechanical contact resistance between the piston and the cylinder is reduced, and the gravity on the piston and the weights is converted accurately into pressure.

The liquid column manometer is a measuring device where the pressure is obtained from the height and density of the liquid column in the U-shaped tube that balances the measured pressure. Normally, the mercury or water with accurately known density is used as the working medium in the U-shaped tube. The foundation of the Japanese national standard for pressure is the primary interferometric standard manometer, which is a liquid column manometer using mercury as the working medium ${ }^{[14]}$. Similar pressure gauges are used as the national standard devices for pressure in the atmospheric pressure region at the national metrology institutes around the world.

The digital pressure gauge is a device that has a pressure sensor and a mechanism to output the result on a display or as digital signals ${ }^{[12]}$. There are several measurement principles of the pressure sensor, and the typical one includes the detection of change in resistance such as the strain gauge, change in capacitance of the capacitance sensor, or change in frequency of the crystal or silicon oscillator. Many are capable of consecutive external output of the measured results as analog or digital signal. With the recent rapid advances in technology, digital pressure gauges with excellent resolution and stability are becoming available.

The mechanical pressure gauge is a measuring device that measures pressure by mechanically expanding the deformation of the elastic element under pressure. The indicator with flat cross-section and bent tube with closed off ends as the elastic elements is called the Bourdon tube pressure gauge. It is used widely in society and industry due to its relatively low $\operatorname{cost}^{[13]}$.

Table 1 is a summary of the characteristics and properties of the four types of pressure gauges. The pressure balance and the liquid column manometer, which are primary pressure gauges, have small uncertainly of measured pressure, excellent long-term stability, and high reliability. However, these pressure gauges, in general, require complex maneuver, are heavy and expensive. Also, the acceleration of gravity of the place of installation is necessary to obtain accurate values for generated pressure. The pressure balance, mechanical pressure gauge, and digital pressure gauge can be used in wide pressure ranges, while the liquid column manometer has limited range compared to the other gauges. The digital pressure gauge and the mechanical pressure gauge that are secondary gauges are lower in reliability compared to the pressure balance and the liquid column manometer, but are easy to handle, light, and are low in cost. Therefore, redundant installation of multiple measuring devices is done to increase the reliability. Also, while the generated pressure of the aforementioned primary gauges changes according to the acceleration of gravity, the measured values of the digital and mechanical pressure gauges are not directly affected by the acceleration of gravity. In general, the digital pressure gauge, in which the continuous measurement and transmission of measured results are easily done, is suitable for automation and systemization, and is useful in increasing the operability and efficiency.

We engaged in the development to utilize the advantages of the digital pressure gauge that are its small and lightweight portability, simple operability, and high applicability to automation and systematization.

\section{R\&D scenario}

In this research, we set the goals as the utilization of the digital pressure gauge to confirm the equivalence of the national standard necessary for international mutual recognition and to increase the reliability of pressure measurement at the industrial sites. 
The research scenario is shown in Fig. 4. The main elemental technologies for this research are the various properties of the digital pressure gauge and the evaluation and calibration technologies. The properties of the digital pressure gauge include the measurement range, resolution, weight, size, linearity, stability in environment change and during transportation, and short- and long-term stability. These properties depend heavily on the manufacturer of the measuring device. On the other hand, the evaluation of various properties and the evaluation of uncertainty of the calibration value for the digital pressure gauge are the technologies in which AIST excels, since it owns the national standard and routinely evaluates various pressure gauges.

To achieve the research objective, the aforementioned elemental technologies are integrated. First, the methods for property evaluation and the calibration methods of the digital pressure gauge are developed, to carefully evaluate the performance of the digital pressure gauge as a standard device. Particularly important are the evaluation technology for the stability of the digital pressures gauge in environment change and during transportation as well as the short- and long-term stability. Next, the use of the digital pressure gauge as the standard device is promoted to further integrate the elemental technologies, and to incorporate it into the metrological standard system including the establishment and maintenance of the national standard for pressure, international comparison of the national standards, and the standard provision in Japan. For the national standard, AIST establishes and manages the standard from $1 \mathrm{~Pa}$ to $1 \mathrm{GPa}$ using multiple standard devices. The technological development is being done to enable efficient, high-precision calibration of the standard devices that are difficult to operate, using the digital pressure gauge effectively. For the international comparison of the national standards, the comparison will be done efficiently using the digital pressure gauge for the major pressure ranges. For provision of the standard in Japan, the standard provision method with least burden to the client will be developed. The pressure calibration service by AIST for digital pressure gauge will be expanded. The standard provision system in Japan is advanced to efficiently provide the pressure standard to society and industry, and various related technology standards will be organized.

Finally, the efficient standard provision using the digital pressure gauge will improve the reliability of pressure measurement at the industrial sites, which is one of the research objectives, in conjunction with the development of the industrial pressure gauge by the measuring device manufacturers. The international comparison using the digital pressure gauge will greatly contribute to the international mutual recognition of pressure measurement, which is the other research objective. We set such a research

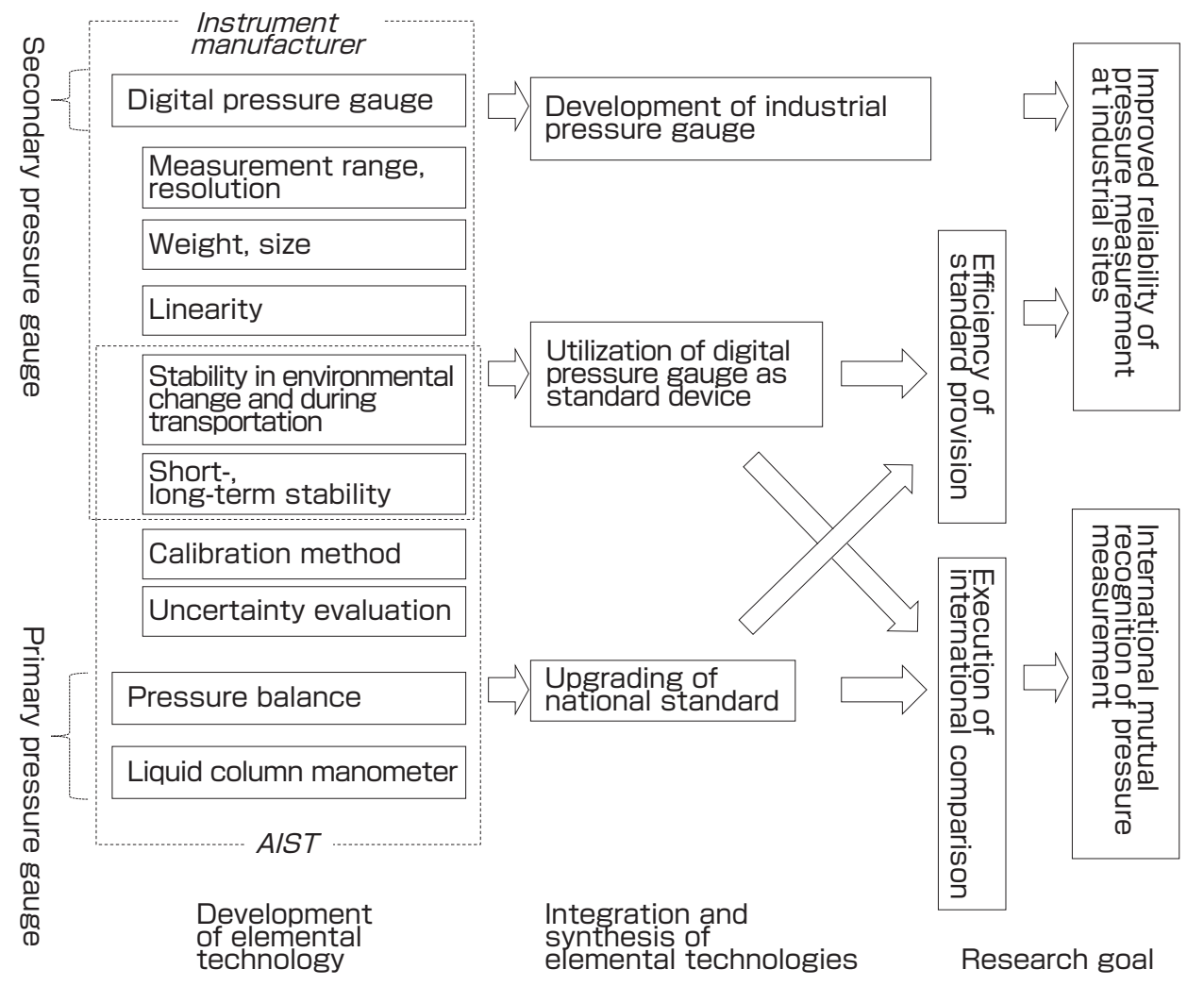

Fig. 4 Research scenario for increasing the reliability of pressure measurement and for international mutual recognition 
scenario. What follows are the contents and results of the R\&D that was conducted according to the scenario.

\section{Evaluation of the properties of the digital pressure gauge}

To build the practical and efficient standard system of pressure while maintaining reliability, the effective use of digital pressure gauge is the key. Therefore, AIST engaged in the R\&D for the property evaluation and calibration method of various digital pressure gauges using the pressure standard devices ${ }^{[15]-[17]}$. The following factors can be given as the main reason for the uncertainty of the measured value displayed by the digital pressure gauge:

a) Uncertainty of the applied pressure

b) Uncertainty due to the variation in measured value

c) Uncertainty due to historical effect

d) Uncertainty due to resolution or short-term stability

e) Uncertainty arising from changes in surrounding temperature (temperature property)

f) Uncertainty arising from attitude of installment (attitude property)

g) Uncertainty due to changes in voltage of power supply

h) Uncertainty due to the linearity of input-output relation (linearity)

i) Uncertainty due to changes in line pressure (in case of differential pressure gauge)

j) Uncertainty arising from long-term stability (change over time)

k) Uncertainty arising from changes in surrounding environment (relative humidity, atmospheric pressure, vibration, impact, etc.)

In case the digital pressure gauge is used as the standard, the above property evaluation must be conducted thoroughly, and consideration is necessary on the uncertainty evaluation and correction of the calibration value according to the situation in which the device is used. Consideration must also be made in transporting the pressure gauge, since the property will be affected by the changes in surrounding environment during transportation.

Currently, various digital pressure gauges with excellent performance are available in wide pressure ranges. The two gauges, for which the evaluation of the property was mainly done in our research, were a differential pressure gauge that uses a silicon resonant sensor as the pressure sensor manufactured by a Japanese measuring instrument company, and a pressure gauge that uses the crystal oscillator as the pressure sensor manufactured by a foreign company. The stability was evaluated by conducting appropriate corrections after performing detailed property evaluation, and then regularly repeating the calibration of the digital pressure gauges using the fixed calibration method. As a result, it was found that reliability equal to the conventional pressure standard device could be obtained for the digital pressure gauge by devising the usage method. The evaluation data for the stability of each pressure gauge is illustrated in the case studies of the incorporation of the digital pressure gauge into the standard system, as will be explained later.

\section{Incorporation of the digital pressure gauge into the standard system}

The three case studies of the incorporation of the digital pressure gauge into the standard system for pressure are described in this chapter.

\subsection{Establishment and maintenance of the national standard}

The liquid column manometer and the pressure balance are positioned as the national standard of Japan or the primary standard of pressure. To use them as the primary standard, it is necessary to determine and manage the various property values. The property values of the pressure standard used at AIST are accurately measured and managed, so they are traceable to the national standards of mass, length, temperature, and others. At AIST, several pressure balances and pistons and cylinders are managed for each respective pressure range. By managing large numbers of standard devices in groups, the national standard is maintained for a wide pressure range. For the maintenance of these standard devices, comparative measurements between the standard devices are conducted regularly to check the mutual conformity. Also, from the results of the comparative measurement for various combinations, the long-term stability of the generated pressure of each standard device is evaluated. Although it depends on the pressure range, in general, the long-term stability is on the relative order of $10^{-6}$ per year.

Here, the case, where the digital pressure gauge was used to calibrate the pressure balance of a private calibration lab with AIST's national standard pressure balance, is described. When the pressure balance is calibrated at AIST, two pressure balances are connected directly to compare the generated pressure. In traditional calibration, the two pressure balances are operated simultaneously, the changes in the fall rate of the pistons are observed, and fine weights are added to either balance until the equilibrium is attained. However, this method involves complex maneuver, and the calibration result is highly dependent on the system configuration and the technical prowess of the calibration operator. Therefore, AIST selected and advanced the two calibration methods using the high resolution and consecutive measurement functions of the digital pressure gauge. As a result, in both methods, it was found that if the performances of the digital pressure gauge used and the pressure balance are good, the equilibrium state could be attained at the 
relative order of $10^{-6}$ or less. Currently, the calibration and maintenance of numerous pressure balances as well as the national standard devices maintained by AIST are done using the digital pressure gauge, and the process has been advanced greatly and can be conducted efficiently.

The first calibration method is the method of directly measuring the generated pressure difference of the two pressure balances using a high-resolution digital differential pressure gauge. In this method, small weights are used to adjust the pressure difference of the two pressure balances measured with the differential pressure gauge until the difference reaches zero.

The second calibration method is the comparative method where the digital pressure gauge is used as the comparator ${ }^{[17][18]}$. As shown in Fig. 5, the generated pressures of the two pressure balances are measured alternately with the digital pressure gauge by switching the constant volume valve. The advantage is that even if there is some degree of difference between the generated pressures of the two pressure balances, the difference can be obtained accurately and corrected. Moreover, this method can be applied simultaneously to three or more pressure balances. In the second calibration method, the short-term stability was utilized in addition to the high resolution and consecutive measurement functions of the digital pressure gauge.

\subsection{International comparison of the national standards}

Normally, in the international comparison of the national standards of pressure, the transfer device, which is a measuring device that can be transported, is circulated among the countries, and the participating national institutes obtain the calibration value for the transfer device based on their own national standards. By mutually comparing the calibration values of each country, the differences of the values of the national standard for pressure of each country can be seen. For the international comparison where the measurement of highest precision is necessary, the pressure

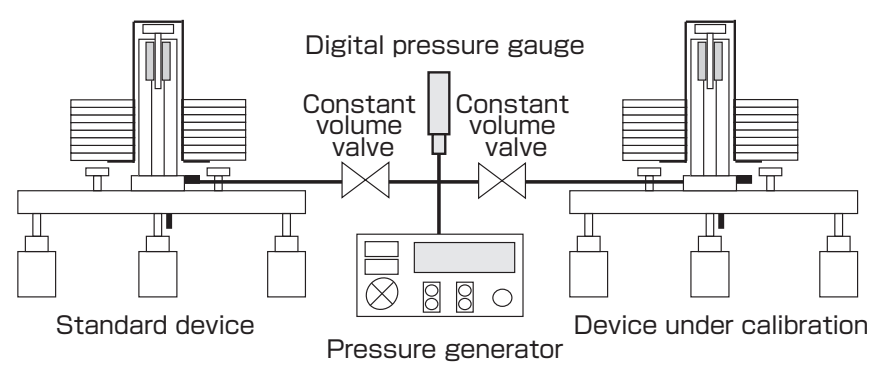

Fig. 5 Calibration by comparative method using the digital pressure gauge as the comparator balance that has equivalent performance as the standard was used traditionally as the transfer device.

In such a situation, AIST focused on a digital pressure gauge that was small, lightweight, and of low cost, as well as easy to handle, and engaged in the world-leading development of a transfer device for international comparison using the high-precision industrial digital pressure gauge. Using the developed transfer device, several international comparisons were actually done ${ }^{[19]-[23]}$. Multiple digital pressure gauges were used simultaneously as the transfer devices, to increase the reliability by redundancy. Through the execution period of the international comparison that normally takes over one year, the details of the long-term property changes of the digital pressure gauge was studied and the results were used to correct the calibration value. By these corrections, the lack of long-term stability of the transfer device was compensated, and sufficient comparative precision could be maintained $^{[19]-[22][24]}$.

As an example of use of the digital pressure gauge in international comparison, we describe the international comparison conducted from 2002 to 2004 to check the international equivalence of the national standard for hydraulic gauge pressure with pressure range from $10 \mathrm{MPa}$ to $100 \mathrm{MPa}$ (APMP.M.P-K $7^{[19]}$ ). Figure 6 shows the transfer device used in that international comparison. The transfer device was composed of a set of two digital pressure gauges with full scale of $100 \mathrm{MPa}$, resolution of $0.1 \mathrm{kPa}$, and used the crystal oscillator as the pressure sensor. With the use of the digital pressure gauge, the total weight of the transfer device during transportation could be kept at $50 \mathrm{~kg}$ or less, and this was dramatic weight reduction compared to using the pressure balance (which usually weighs over $200 \mathrm{~kg}$ ). Also, three sets of transfer devices with the same specifications were prepared and sent simultaneously to the participating institutes by

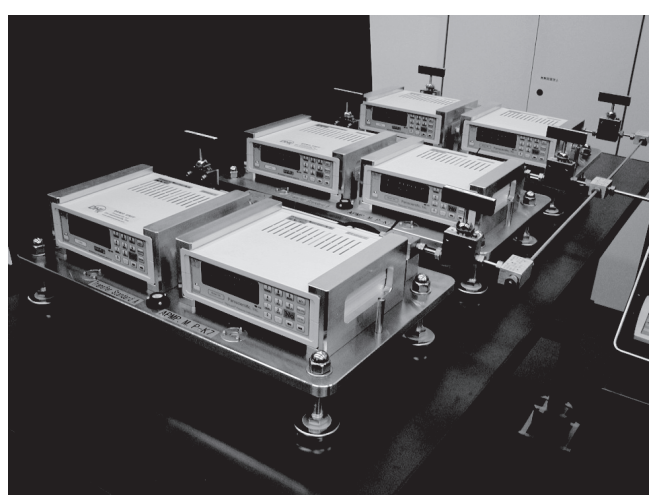

Fig. 6 Photographs of the transfer device for international comparison for hydraulic pressure using the digital pressure gauge (two pressure gauges on each transfer device, total three sets) 
different circulation routes to greatly reduce the measurement period required for the international comparison.

During the measurement period, a total of six digital pressure gauges that comprised the transfer device were regularly returned to AIST that acted as the pilot institute. The stability of the transfer device was evaluated by recalibrating these digital pressures gauges using the Japanese national standard. Figure 7 shows the change per day of the calibration values of each digital pressure gauge, as the evaluation of the function of measured values. As seen in the graph, the change at the maximum measured pressure of $100 \mathrm{MPa}$ was over $50 \mathrm{~Pa}$ per day for some pressure gauges. Since there were about 400 days for the entire measurement period of the international comparison, the measured pressure value at the maximum pressure was estimated to change $20 \mathrm{kPa}$. This corresponds to relative change of $2 \times 10^{-4}$ against the measured value. On the other hand, when the pressure balance that was traditionally used as the transfer device in the international comparison was used in this pressure range, the stability was expected to be about relative $1 \times 10^{-5}$. Therefore, when the above digital pressure gauge was used without correcting the calibration value, the stability necessary for the international comparison could not be obtained. However, it was found in the evaluation that the change in each pressure was the linear function of the number of lapsed days. By correcting this and by using the average value of the calibration values obtained from the two digital pressure gauges that comprised the transfer device, ultimately, $5 \times 10^{-6}$ or less for the stability of each transfer device was obtained throughout the entire measurement period ${ }^{[19]}$. We were able to obtain sufficient comparative precision that could be used in the international comparison, by understanding the details of the property of the digital pressure gauge as the transfer device and conducting appropriate corrections.

Figure 8 shows the results of the calibration values at 100 $\mathrm{MPa}$ of the participating institutes in the two international

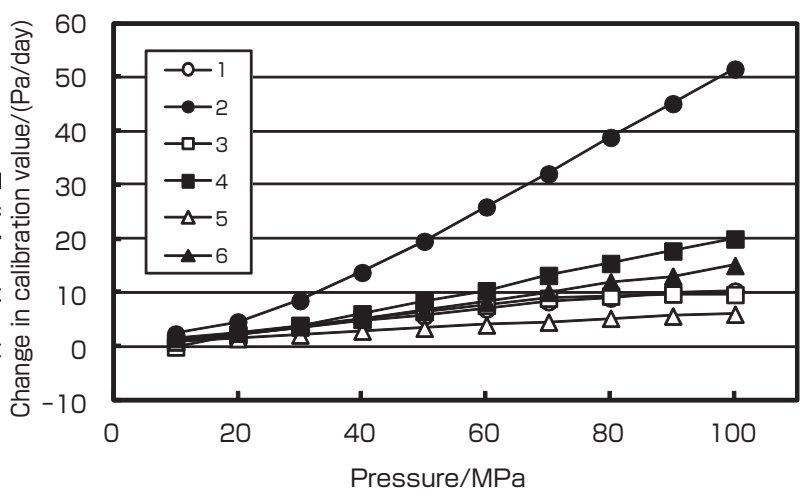

Fig. 7 Changes per day of the calibration value of the six digital pressure gauges used for the international comparison of hydraulic pressure comparisons (CCM.P-K $7^{[25]}$ and APMP.M.P-K $7^{[19]}$ ), including the aforementioned international comparisons. The horizontal axis shows the acronyms of the participating institutes and the country names, and the vertical axis shows the relative deviation from the international comparison reference value of the calibration values of each institute. The reference value was obtained by calculation of the average value of the calibration values of the participating institutes. The length of the bar for the data for each country is the uncertainty (confidence level about $95 \%$ ) of the calibration value claimed by the participating institutes. The results obtained in the same international comparison are indicated by the same markers (black or white circles). The equivalence of the national standards for pressure of the countries can be seen from Fig. 8. The Japanese national standard has almost zero deviation from the reference value, and the uncertainty is similar to the major countries. Therefore, it was confirmed that the Japanese national standard for pressure established, maintained, and supplied for the target pressure range has excellent international equivalence.

The use of digital pressure gauge as the transfer device is being done in other international comparisons. Excellent comparative precision was obtained by using the digital pressure gauge with silicon resonant sensor in the international comparison of the gas differential pressure ${ }^{[20]}$. In this manner, the efficacy of the digital pressure gauge as the transfer device was confirmed in the international comparison in various pressure ranges.

\subsection{Standard provision through remote calibration}

In the calibration of pressure gauges used in the industrial sites, the "carry-in calibration" is the common practice where

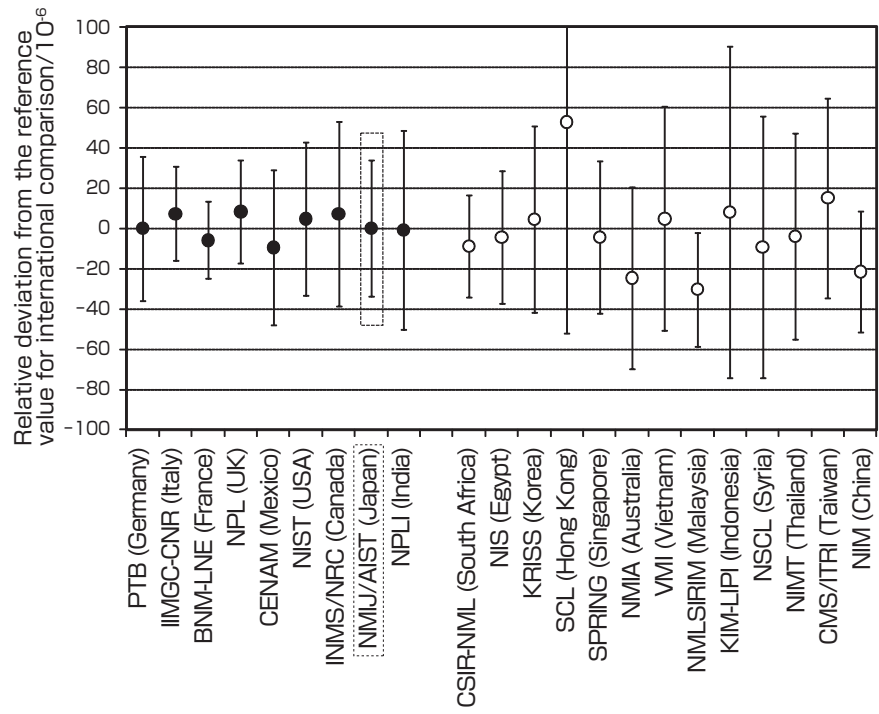

Fig. 8 Results of the two international comparisons at pressure $100 \mathrm{MPa}$

(O:CCM.P-K7, O:APMP.M.P-K7) 
Table 2. Form and characteristic of the calibration of pressure gauge

\begin{tabular}{l|lll}
\hline Calibration form & Carry-in calibration & $\begin{array}{l}\text { Dispatch calibration } \\
\text { (on-site calibration) }\end{array}$ & Remote calibration \\
\hline Place of calibration & Calibration lab & $\begin{array}{l}\text { Client-designated } \\
\text { place }\end{array}$ & $\begin{array}{l}\text { Client-designated } \\
\text { place }\end{array}$ \\
\hline Standard device & Reference standard & $\begin{array}{l}\text { Transfer device } \\
\text { (transfer standard) }\end{array}$ & $\begin{array}{l}\text { Transfer device } \\
\text { (transfer standard) }\end{array}$ \\
\hline Calibration work & Lab personnel & Lab personnel & $\begin{array}{l}\text { Remote operation, } \\
\text { support personnel }\end{array}$ \\
\hline
\end{tabular}

the client takes the pressure gauge from the usage site to the calibration lab. However, for the pressure standard to be used in a wider range of industrial fields, it is necessary to consider a more efficient, new ways of providing the standard. In the NEDO Project "Technology Development for an 'e-trace' System", the remote calibration technology for pressure was developed to provide the pressure standard quickly, at low cost, and accurately, by utilizing the digital pressure gauge and the information technology such as the Internet ${ }^{[26][27]}$. This was a technological development that allowed the calibration of the pressure gauge at the industrial sites without moving it from the industrial site to the far away calibration lab, and was a new form of providing the pressure standard to reduce the burden on the calibration client.

Currently, when the calibration labs conduct calibration by request from the clients, the calibration can be roughly divided into three forms: carry-in calibration, dispatch calibration, and remote calibration. The major characteristics of each calibration form are shown in Table 2.

In the traditional carry-in calibration, the calibration is done as the client brings the pressure gauge to the calibration lab. In this case, the standard device of the calibration lab is used, and the work is done by the personnel of the calibration lab. When it is impractical to transport the client's pressure gauge to the lab, the calibration is done at the place where the pressure gauge is installed. The calibration done outside of the calibration lab is the dispatch (on-site) calibration. In this case, the transportable standard device (transfer device) is transported from the calibration lab to the site. The calibration work is done by the personnel of the calibration lab, as in the case of carry-in calibration.

In remote calibration, calibration is done by sending the transfer device to the place where the client's pressure gauge is being used, as in the dispatch calibration. However, the calibration lab personnel does not go to the site, and the calibration work is done by exchanging the measurement data via communication technology such as the Internet from the calibration lab. This point is different from the dispatch calibration. It is assumed that the client's pressure gauge (device under calibration) is a digital pressure gauge.
The supporting personnel at the client company attends a technical seminar to do the work of installing the device under calibration and the transfer device, as this will not greatly affect the calibration result.

Figure 9 shows the procedure for remote calibration of pressure. It shows an example of the process where the client makes a request for remote calibration to the calibration lab. In this figure, the area surrounded by dashed line and marked "Internet" is the part that can be accomplished via the information network technology.

The development of the remote calibration technology was done for the two pressure ranges (gas differential pressure of 10 $\mathrm{Pa} \sim 10 \mathrm{kPa}$ and hydraulic pressure of $10 \mathrm{MPa} \sim 100 \mathrm{MPa}$ ) for which the performance of the digital pressure gauge has been proven in the past international comparisons, and where there was high demand for calibration from industry. Technological collaborations were obtained from Yokogawa Electric Corporation for the development of gas differential pressure, and Nagano Keiki Co., Ltd. for hydraulic pressure. The main items of development were as follows:

(1) Development of transfer device for remote calibration

(2) Development of measurement procedure applicable for remote calibration

(3) Execution of demonstration experiment in Japan and overseas

For Item (1), the portable transfer device for remote calibration was newly developed by incorporating the high-precision digital pressure gauge, pressure generator, and environmental

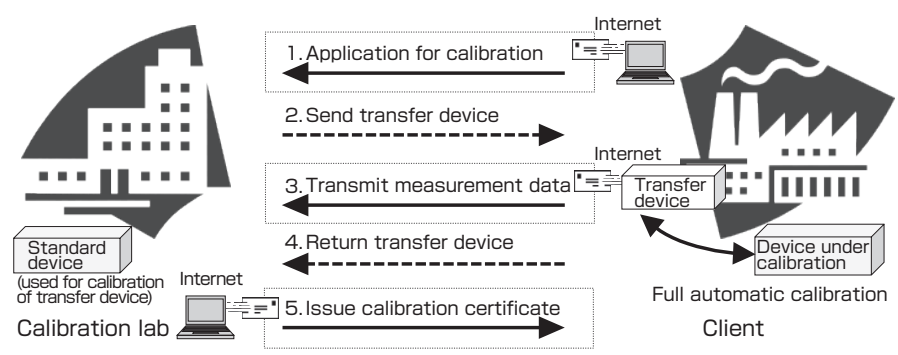

Fig. 9 Execution procedure for remote calibration for pressure 
measuring device into one body to enable the fully automatic pressure generation and measurement. To increase the reliability of the transfer device, multiple high-precision digital pressure gauges were installed in each transfer device. For the gas differential transfer device, the pressure gauge with silicon resonant sensor as the pressure sensor was used, and for the hydraulic pressure transfer device, the digital pressure gauge with crystal oscillator as the pressure sensor was employed. To compensate for the lack of long-term stability of the installed digital pressures gauges, the detailed preliminary studies were done for the properties such as the stability in environment change and during transportation as well as the short- and long-term stability.

For Item (2), the program for conducting automatic calibration was created. Also, the remote calibration manuals for the personnel at the calibration lab and the supporting personnel at the client company were drafted.

For Item (3), numerous demonstration experiments for remote calibration were done in Japan and abroad using the developed transfer device. Figure 10 shows an example of the demonstration experiment for the gas differential pressure. As shown in Fig. 10(a), the remote calibration experiment was done by sending the transfer device twice to Kofu, Yamanashi Prefecture and once to Chongqing, China, with AIST as the calibration lab. Figure 10(b) shows the results of calibration of the digital pressure gauge with full scale of $10 \mathrm{kPa}$ and resolution of $10 \mathrm{mPa}$, and they are shown as the deviation from the calibration value obtained in ordinary carry-in calibration. The maximum value of the deviation of each measured pressure was within $20 \mathrm{mPa}$, and this was sufficiently small at relative $2 \times 10^{-6}$ or less of the full scale of calibrated pressure gauge. From this result, it was shown that the result equivalent to the carry-in calibration could be obtained by remote calibration, and that sufficient stability including the stability in environment change and during transportation could be obtained for the transfer device and calibrated digital pressure gauge.

The newly developed remote calibration technology was confirmed to be reliable from the results of the demonstration experiment, and it is currently established as the calibration service at AIST. The current uncertainties (confidence level of about $95 \%$ ) for the remote calibration service are $100 \mathrm{mPa}$ or $0.01 \%$ or less for gas differential pressure in the range from 10 Pa to $10 \mathrm{kPa}$, and $0.01 \%$ or less for hydraulic pressure in the range from $10 \mathrm{MPa}$ to $100 \mathrm{MPa}$.

\section{Ripple effect to society and industry, and future issues}

\subsection{Increasing the reliability of pressure measurement at industrial sites}

Currently, AIST establishes the national standards for the nine-digit pressure range from $1 \mathrm{~Pa}$ to $1 \mathrm{GPa}$, and responds to the various calibration requests from society and industry. For the maintenance and advancement of the group of pressure standard devices that comprise the national standard for pressure, the use of digital pressure gauge is promoted, as described in subchapter 6.1. Currently, we are developing the full automatic calibration technology for multiple pressure balances using the digital pressure gauge. This is expected to enhance the advancement and efficiency of the group-managed national standard devices.

For the main part of the calibration service currently conducted by AIST, the conformity assessment has been completed for ISO/IEC17025, which is the international standard that is the general requirement for the testing and calibration labs, as well as the technical assessment by the assessment personnel who are specialists invited from major overseas national metrology institutes. AIST's ability for calibration and measurement are recognized by the rest of the world.

The pressure standard provision to the industrial measuring sites and Japanese users is done mainly by the calibration labs for pressure accredited by the Japan Calibration Service

(a)

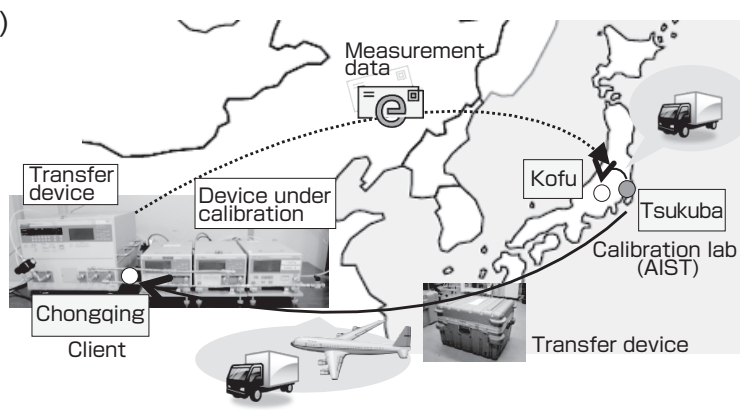

(b)

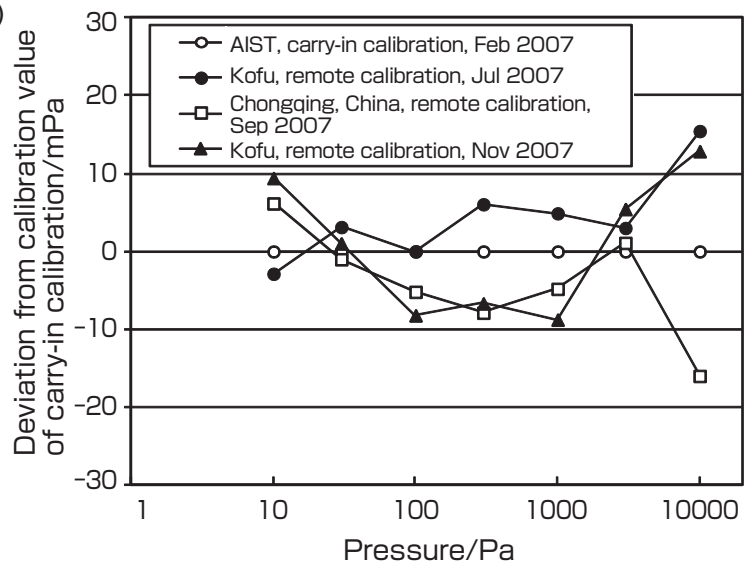

Fig. 10 (a) Circulation route of the transfer device in the demonstration experiment for the remote calibration of gas differential pressure and (b) the results (the calibration value of the remote calibration was expressed as the deviation from the calibration value of carry-in calibration) 
System (JCSS) based on the Measurement Laws. Figure 11 shows the outline of this system. The values of the national standard are provided in the major pressure ranges for gas and hydraulic pressures, by AIST calibrating the pressure balances of the JCSS calibration lab. The JCSS calibration labs owns the standard device traceable to the AIST's national standard, builds the quality system through common requirements and application guideline, and are assessed by the accreditation organization. The JCSS calibration lab calibrates the various industrial pressure gauges under the JCSS using their own standard devices. AIST engages in the creation of the technical standard for pressure for JCSS, and also supports and cooperates in the accreditation assessment from the technical standpoint.

Currently, the list of accredited calibration labs and their best measurement capability can be seen under JCSS, Conformity Assessment in the website of the National Institute of Technology and Evaluation (NITE) (http://www.nite.go.jp/). As of September 2011, there are 13 calibration labs accredited in the "Pressure" section of JCSS. The number of JCSS certificates issued by the calibration labs has increased in the past three years, and about 2,300 certificates were issued in FY 2010. The number of certificates issued is expected to increase further in the future. The importance of the pressure measurement traceable to the national standard is increasing in the Japanese society and industry. The types of pressure gauge most calibrated are the digital pressure gauge and the mechanical pressure gauge. As mentioned earlier, the measured values of such pressure gauges are not directly affected by the acceleration of gravity, hence they do not have to be corrected and the traceability can be maintained easily. Also, the pressure balance that is normally used as the

AIST

\section{National standard for pressure}

Primary interferometric standard manometer (liquid column manometer) Primary pressure standard device group (pressure balance)

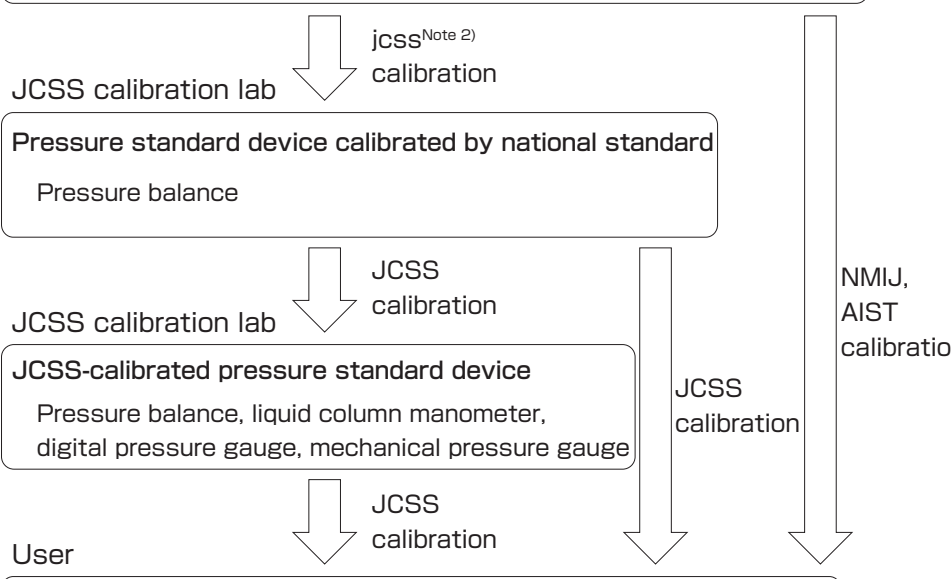

General pressure measuring device on site

Pressure balance, liquid column manometer, digital pressure gauge, mechanical pressure gauge

Fig. 11 Japanese standard provision system for pressure standard device in the high-pressure range tends to be large, and this may be a major burden on the calibration lab and the user for its installation and maintenance. However, the burden can be reduced by using the digital pressure gauge as the standard device.

While it is not discussed in this paper in detail, the organization of the differential pressure standard has progressed recently. There is a system where the JCSS calibration labs can set the differential pressure standard themselves using two JCSS calibrated pressure balances ${ }^{[8][15]}$. While the method using the two pressure balances ensures high precision, it is not a simple method that can be use in daily calibration work. Therefore, we developed the standard provision system using the digital differential pressure gauge to widely and efficiently disseminate the differential pressure standard. The calibration of the digital differential pressure gauge by the JCSS calibration labs has been available since 2008 , and it has become possible to efficiently provide the differential pressure standard.

AIST has cooperated as the technical experts to organize various industrial standards ${ }^{[11]-[13]}$ and technical standards, to enable the users of the pressure gauge and industry to utilize the technologies and findings pertaining to the property evaluation of the digital pressure gauge and the calibration method obtained by R\&D. Particularly, procedures of characterization and calibration for digital pressure gauges were set in JIS B $7547^{[12]}$.

The calibration method and the technology using the digital pressure gauge are improving the reliability of the pressure measurement at industrial sites through the AIST's calibration service, Japanese standard system, and various measurement and technical standards. In the development described in this paper, the main focus was on the use of relatively high precision digital pressure gauge. In the future, to maintain the reliability of pressure measurement efficiently and at low cost, it is necessary to apply the developed property evaluation and the calibration method to general industrial digital pressure gauge, and to incorporate them into the standard system.

The remote calibration is a technical development to reduce the burden on the client and to conduct the standard provision quickly, at low cost, and accurately. In this $R \& D$, the basic technology necessary for remote calibration for pressure, or the technology to conduct calibration in a short time while maintaining reliability at the industrial site was developed. However, since the manufacturing cost of the transfer device for remote calibration is not kept low, currently the calibration cost is not lower than the carry-in calibration, and this is one factor that inhibits its wide use. Also, the framework for accrediting the calibration lab that conducts remote calibration is an issue. In the future, we would like to promote the use of 
Table 3. International comparison for pressure in which AIST participated (since 2001)

\begin{tabular}{l|lll}
\hline Identifier & Kind & Pressure range & Year executed \\
\hline APMP.M.P-K6 & Gas gauge pressure & $20 \mathrm{kPa}-105 \mathrm{kPa}$ & $1998-2001$ \\
\hline APMP.M.P-K1.C & Gas gauge pressure & $0.4 \mathrm{MPa}-4 \mathrm{MPa}$ & $1998-2001$ \\
\hline APMP.M.P-K9 & Gas absolute pressure & $10 \mathrm{kPa}-110 \mathrm{kPa}$ & $2009-$ \\
\hline APMP.M.P-K5* & Gas differential pressure & $1 \mathrm{~Pa}-5 \mathrm{kPa}$ & $2005-2006$ \\
\hline CCM.P-K7 & Hydraulic gauge pressure & $10 \mathrm{MPa}-100 \mathrm{MPa}$ & $2003-2005$ \\
\hline APMP.M.P-K7* & Hydraulic gauge pressure & $10 \mathrm{MPa}-100 \mathrm{MPa}$ & $2002-2005$ \\
\hline APMP.M.P-K7.1* & Hydraulic gauge pressure & $10 \mathrm{MPa}-100 \mathrm{MPa}$ & $2007-2009$ \\
\hline APMP.M.P-K7.TR/* & Hydraulic gauge pressure & $40 \mathrm{MPa}-200 \mathrm{MPa}$ & 2001 \\
\hline CCM.P-K13 & Hydraulic gauge pressure & $50 \mathrm{MPa}-500 \mathrm{MPa}$ & $2008-2011$ \\
\hline APMP.M.P-K13* & Hydraulic gauge pressure & $50 \mathrm{MPa}-500 \mathrm{MPa}$ & $2010-$ \\
\hline APMP.M.P-S8* & Hydraulic gauge pressure & $100 \mathrm{MPa}-1000 \mathrm{MPa}$ & $2007-2010$ \\
\hline
\end{tabular}

*International comparison with AIST as the pilot institute

related technologies for the pressure gauge calibration at the industrial sites by the calibration labs.

\subsection{International mutual recognition of pressure measurement}

AIST participated in the international comparison of the national standards for pressure conducted by the Comite Consultatif pour la Masse et les Grandeurs Apparentées (CCM) of CIPM, as well as the Consultative Committee for Mass of the Asia Pacific Metrology Program (APMP) ${ }^{[19]-[23][25]}$. Table 3 shows the international comparisons in which AIST had participated and the ones in progress. AIST organized the international comparison as the pilot institute in the majority of the APMP international comparisons, and engaged in the creation of the execution schedule, drafting of the measurement manual, preparation of the transfer device and the property evaluation, organization of the comparison results, and the write-up of the final repor ${ }^{[19]-[22]}$. In the international comparisons organized by AIST, the digital pressure gauge was used actively as the transfer device to enhance efficiency, as mentioned in subchapter 6.2.

The international equivalence of the AIST national standard was confirmed according to the results of the international comparisons of several pressure ranges ${ }^{[19]-[23][25]}$. AIST is planning to participate actively in the future international comparisons, and will continue to ensure the international equivalence of the Japanese national standards.

The results of the international comparisons conducted by AIST as the pilot institute are registered in the Appendix B of the international Mutual Recognition Arrangement, CIPM, as mentioned in subchapter 2.2. The calibration and measurement capabilities that the participating national metrology institutes claimed based on the results of the international comparison are registered in the Appendix C of the Mutual Recognition Arrangement after international recognition. By actively participating in the international comparison activities, we believe we were able to support the smooth accreditation of the calibration and measurement activities of several countries, mainly of Asia, as well as Japan.

By utilizing the digital pressure gauge, we were able to reduce the size and weight of the transfer device, hence reducing the problems during transportation. We were also able to obtain sufficient comparative precision by evaluating the long-term stability using multiple digital pressure gauges for redundancy in each transfer device. Also, the international comparison could be done in short periods by preparing multiple transfer devices and circulating them simultaneously. By using the transfer device comprised of the digital pressure gauge in the international comparison, we were able to efficiently confirm the international equivalence of the national standard necessary for international mutual recognition.

\section{Conclusion}

The R\&D was conducted to confirm the international equivalence of the national standard for the international mutual recognition and to maintain the reliability of the pressure measurement at the industrial site, using the digital pressure gauge. As the pressure standard at the industrial site, the pressure balance and the liquid column manometer that have excellent long-term stability were traditionally used, but the use of the digital pressure gauge and various pressure generators are now increasing. The technologies for property evaluation and calibration of the digital pressure gauge described in this paper are used effectively in the pressure standard system, and the practical application of the effective standard provision system using the digital pressure gauge as the standard or transfer device is progressing. With the diffusion of JCSS, the calibration of the digital pressure gauge traceable to the national standard for pressure for a wide pressure range is now available to the general user through accredited pressure calibration labs, and this contributes to the increased reliability of onsite pressure measurement. 
In the highly advanced and diversified social and industrial activities, the ensuring of reliability of pressure measurement according to usage and precision are demanded. The development of pressure calibration and standard provision that are easier, faster, and lower in cost, as well as being practical and efficient, are important, taking advantage of the operability and convenience of the digital pressure gauge in the future.

We shall engage in further R\&D to maintain and improve the reliability of various pressure measurements in society and the industrial sites, by organizing and expanding the pressure standard provision system.

\section{Acknowledgements}

I am deeply grateful to Dr. Akira Ooiwa, head of Mechanical Metrology Division, National Metrology Institute of Japan, who gave us valuable advice and cooperation, and the people of the Pressure and Vacuum Standard Section.

\section{Notes}

Note 1) The deadweight pressure balance or deadweight pressure gauge is commonly called "pressure balance", "piston gauge", or "deadweight tester" in English. In this paper, it will be called the "pressure balance" which is the term generally used in the technical documents for various standards including JIS.

Note 2) In the Japan Calibration Service System under the Measurement Laws, the lower case letters "jcss" is used in the calibration certificate issued by the national metrology institute, and the upper case letters "JCSS" is used on the calibration certificate issued by the accredited calibration labs.

\section{References}

[1] A. Ono: Globalizing metrological standards, AIST Today, 4 (2), 21 (2004) (in Japanese).

[2] M. Tanaka: Innovation and metrology, AIST Today, 10 (12), 2 (2010) (in Japanese).

[3] CIPM, Mutual recognition of national measurement standards and of calibration and measurement certificates issued by national metrology institutes (MRA), (1999).

[4] A. Ooiwa: Mass and related quantities; Mechanical metrology, Journal of the Society of Instrument and Control Engineers, 48 (4), 313-320 (2009) (in Japanese).

[5] T. Kobata: Development, maintenance and dissemination of pressure standard and improvement of reliability, Netsu Sokutei, 38 (2), 57-64 (2011) (in Japanese).

[6] Pressure and Vacuum Standards Section: R\&D of pressure and vacuum standards and calibration service, Journal of the Japan Society of Precision Engineering, 77 (8), 755-756 (2011) (in Japanese).
[7] Heisei 14 Nendo Butsuri Hyojun Nizu Chosa Hokokusho (Survey Report for Physical Standard Demand FY 2002), National Metrology Institute of Japan, AIST (2003) (in Japanese).

[8] M. Kojima, T. Kobata. K. Saitou and M. Hirata: Development of small different pressure standard using double pressure balances, Metrologia, 42 (6), S227-S230 (2005).

[9] T. Kobata and K. Ide: Development of pressure standard up to $1 \mathrm{GPa}$ using a precise pressure multiplier, Proceedings of SICE-ICASE International Joint Conference 2006, 3367-3371 (2006).

[10] H. Kajikawa, T. Kobata and A. Ooiwa: Features of a new controlled-clearance pressure balance and in situ mass calibration of its weights, Trans. of the Society of Instrument and Control Engineers, 44 (3), 219-226 (2008).

[11] JIS B 7610-1, -2, -3, Pressure balances (2000) (in Japanese).

[12] JIS B 7547, Procedures of characterization and calibration for digital pressure gauges (2008) (in Japanese).

[13] JIS B 7505-1, Aneroid pressure gauges - Part 1: Bourdon tube pressure gauge (2007) (in Japanese).

[14] A. Ooiwa, M. Ueki and R. Kaneda: New mercury interferometric baromanometer as the primary pressure standard of Japan, Metrologia, 30 (6), 565-570 (1994).

[15] M. Kojima. T. Kobata and K. Saitou: Study on calibration procedure for differential pressure transducers, Proceedings of IMEKO 20th TC3, 3rd TC16 and 1st TC22 International Conference, ID-044 (2007).

[16] H. Kajikawa and T. Kobata: Effects of pressurization procedures on calibration results for precise pressure transducers, Meas. Sci. Technol., 21 (6), 065104 (2010).

[17] T. Kobata and D. A. Olson: Accurate determination of equilibrium state between two pressure balances using a pressure transducer, Metrologia, 42 (6), S231-S234 (2005).

[18] T. Kobata: Improved methods for comparing gas and hydraulic pressure balances, Metrologia, 46 (5), 591-598 (2009).

[19] T. Kobata et al.: Final report on key comparison APMP. M.P-K7 in hydraulic pressure from $10 \mathrm{MPa}$ to $100 \mathrm{MPa}$, Metrologia, 42 Tech. Suppl., 07006 (2005).

[20] T. Kobata et al:: Final report on key comparison APMP. M.P-K5 in differential pressure from $1 \mathrm{~Pa}$ to $5000 \mathrm{~Pa}$, Metrologia, 44 Tech. Suppl., 07001 (2007).

[21] T. Kobata et al.: Final report on key comparison APMP. M.P-K7.1 in hydraulic gauge pressure from $10 \mathrm{MPa}$ to 100 MPa, Metrologia, 46 Tech. Suppl., 07008 (2009).

[22] T. Kobata et al.: Final report on supplementary comparison APMP.M.P-S8 in hydraulic gauge pressure from $100 \mathrm{MPa}$ to 1000 MPa, Metrologia, 47 Tech. Suppl., 07009 (2009).

[23] T. Kobata: Assurance of the international conformity of pressure standard-International comparison of hydraulic pressure standard from $10 \mathrm{MPa}$ to100 MPa, AIST Today, 6 (5), 34-35 (2006) (in Japanese).

[24] T. Kobata: Characterization of quartz Bourdon-type highpressure transducers, Metrologia, 42 (6), S235-S238 (2005).

[25] W. Sabuga et al.: Final report on key comparison CCM. P-K7 in the range $10 \mathrm{MPa}$ to $100 \mathrm{MPa}$ of hydraulic gauge pressure, Metrologia, 42 Tech. Suppl., 07005 (2005).

[26] M. Kojima, H. Kajikawa and T. Kobata: Development of remote calibration technology for digital pressure gauge, Measurement Standards and Metrology Management, 58 (4), 56-61 (2009) (in Japanese).

[27] T. Kobata: Full Research in pressure standard that supports the social infrastructure-Upgrading and dissemination of the pressure standard and the development of new calibration technology, AIST Today, 10 (1), 18-19 (2010) (in Japanese). 


\section{Authors}

\section{Tokihiko KoBATA}

Completed the doctorate courses at the Department of Physical Engineering, Graduate School of Engineering, Tsukuba University in 1995. Doctor (Engineering). Joined the National Research Laboratory of Metrology, Agency of Industrial Science and Technology, Ministry of International Trade and Industry in 1995. Senior researcher in 1999. Senior

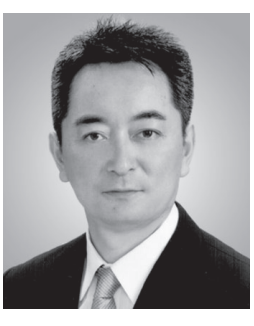
researcher of the Pressure and Vacuum Standard Section, Mechanical Metrology Division, National Metrology Institute of Japan, AIST in 2000. Section chief in 2011 to present. Visiting researcher of the National Institute of Standards Technology, USA from 2000 to 2001. Senior researcher of the R\&D Technological Development Promotion Department, New Energy and Industrial Technology Development Organization from 2008 to 2009. Engages in research for pressure standard and pressure measurement. For this paper, was in charge of the organization of the research, R\&D and calibration work for pressure in general, and drafting of the manuscript.

\section{Momoko KoJima}

Completed the doctorate course at the Department of Energy Sciences, Interdisciplinary Graduate School of Science and Engineering, Tokyo Institute of Technology in 2003. Doctor (Science). Joined AIST in 2003. Researcher of the Pressure and Vacuum Standard Section, Mechanical Metrology Division, National Metrology Institute of Japan, AIST.

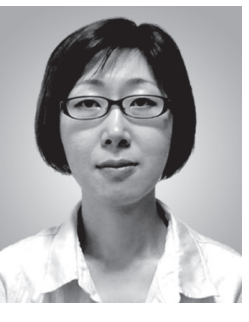
Engages in research for pressure standard and pressure measurement. For this paper, was in charge of the R\&D and calibration work for gas pressure in general.

\section{Hiroaki KAJIKAWA}

Withdrew from the doctorate course with the completion of course requirements at the Division of Physics and Astronomy, Graduate School of Science, Kyoto University in 2006. Joined AIST in 2006. Doctor (Science). Researcher of the Pressure and Vacuum Standard Section, Mechanical Metrology Division, National Metrology Institute of Japan, AIST. Engages in research for pressure standard and pressure measurement. For this paper, was in charge of the R\&D and calibration work for hydraulic pressure in general.

\section{Discussions with Reviewers}

\section{General}

Comment (Akira Ono, AIST)

This paper describes an excellent research to improve the reliability in pressure measurements at industrial environments and to promote the international mutual recognition of pressure measurement results, by adeptly incorporating industrial digital pressure gauges with increased precision into the national standards system. Processes to identify elemental technologies by looking at the overall standards system for pressure measurements, and then creating a new pressure standards system by integrating the elemental technologies are well described. I think it is an appropriate Synthesiology paper.

\section{Cause of time-dependent change of digital pressure gauge Question (Akira Ono)}

Figure 7 in the text shows change with time in the calibration results of digital pressure gauges used for the international comparison. While it may be difficult, since the degree of timedependent change differs for individual pressure gauges, do you have any idea of what caused the time-dependent change?

Answer (Tokihiko Kobata)

The degrees of time-dependent change of the calibration value of the pressure gauge differ according to the types of gauges, the pressure range in which it is used, and the way it is used. In general, the timedependent change of the calibration value of the pressure gauge is large in the gauge for high pressure, and small in the one for low pressure. In some cases, time-dependent change may not be so apparent. Also, as shown in Fig. 7, the amount of time-dependent change may be the function of applied pressure within a pressure gauge.

Therefore, while I cannot generalize, one factor for timedependent change of the calibration value is the effect of plastic deformation of the pressure receiver. If the pressure receiver undergoes complete elastic deformation, it deforms according to the amount of pressure applied, and returns to the original form when the pressure becomes zero. However, in actual pressure gauges, when the pressure is applied, the pressure receiver may undergo slight plastic deformation. Even though the deformation may be slight, it will not return to the original shape completely when the pressure is back to zero. When the process of applying pressure then returning to zero is repeated, the receiver shows larger deformation with the same pressure. In the example of Fig. 7 , the calibration values of the six pressure gauges all change as the deformation of the receiver increases with time, even at the same applied pressure. The time-dependent change of these calibration values contains the effect of the plastic deformation as described.

However, such changes are very small compared to the specified precision stated by the manufacturers, and it is hardly a problem in general use. However, in international comparison, the change must be corrected since a high level of measurement precision is demanded.

\section{Quantitative comparison of the conventional method and the new method}

Question (Jun Hama, Energy Technology Research Institute, AIST)

The calibration technology, where the high-precision digital pressure gauge is used as the transfer device, greatly contributes in reducing the works for the on-site pressure calibration, as a means to guarantee data reliability in product development and R\&D.

Please indicate the actual cases where there were reductions of number of days or cost of calibration compared to the conventional method, due to the easier, more efficient, and lower cost of on-site calibration.

\section{Answer (Tokihiko Kobata)}

As the actual case of efficiency, normally one to two weeks of calibration period is needed, including the transportation of the device under calibration, in the carry-in calibration for the digital pressure gauge, while in the various demonstration experiments conducted in Japan, the calibration could be done in two days by remote calibration in most cases. Also, in remote calibration, the duty hours of the person in charge of the calibration can be reduced, so the cost of labor can be reduced. However, currently, 
the cost of remote calibration is not sufficiently low compared to carry-in calibration. The main reason is because the cost of the multi-function, high-performance transfer device used for remote calibration is high. To diffuse remote calibration, it is necessary to decrease the cost of the transfer device by narrowing down the functions according to the required uncertainty.

\section{Region where the digital pressure gauge can be used as transfer device \\ Question (Jun Hama)}

Please describe the specific work on the development of the simple, fast, and low cost method for calibration and standard provision for pressure using the digital pressure gauge, as much as you can disclose. Particularly, the advancement of remote calibration method at the industrial sites is an important key word. What is your assumed region of coverage in Japan and abroad? Are there some regions that are limited due to the uncertainty factors of the digital pressure gauge?

\section{Answer (Tokihiko Kobata)}

The specific developments were added in subchapter 6.3 for the remote calibration of pressure. I think it will become important to provide the realized values of the national standard for pressure widely and smoothly to the industrial sites. In the future, to further increase the reliability of pressure measurement, I think it is necessary to not only guarantee the results of calibration done at intervals but also to guarantee the reliability of the displayed values of the pressure gauge used in the industrial sites consecutively during regular use.

For the region covered by remote calibration, basically if it is a place where the transfer device can be sent safely, installed stably, and where the information network such as the Internet can be used, I don't think there is any particular limitation, regardless of whether it is in Japan or abroad. However, in regions that may require a long time to send the transfer device, quick calibration that is the merit of remote calibration may be lost. Also, as you indicated, since the digital pressure gauge is used as the transfer device in remote calibration, the uncertainty factors shown in chapter 5 must be considered according to the environment where the calibration is done. For example, if the air conditioning is not sufficient, the uncertainty of calibration may increase due to the changes in the surrounding temperature.

\section{Trends of foreign countries in using the digital pressure gauge \\ Question (Akira Ono)}

Is there a trend toward incorporation of high-precision digital pressure gauges as a transfer device in foreign countries as well? Or is it a trend only in Japan?

Answer (Tokihiko Kobata)

In this paper, the establishment and maintenance of the national standard for pressure, international comparison of the national standard, and the cases for incorporating the digital pressure gauge into the Japanese national standard were described. I shall explain the situations.

First, on the use of digital pressure gauge in the comparative measurement of the pressure balance used for the establishment and maintenance of the national standard for pressure, the method of using the digital differential pressure gauge or the first calibration method described in subchapter 6.1 is used in other countries particularly for the calibration of gas pressure. However, the comparative method, the second calibration method, includes an elemental technology developed originally by AIST, and currently only AIST applies this method to the calibration in a wide range of pressure from low to $1 \mathrm{GPa}$. These two methods upgraded by AIST are recognized to conform sufficiently to the calibration results of the traditional methods, and are suitable for automation. In fact, they are being employed by Japanese calibration labs, and I believe it will eventually be used widely in other countries also.

Next, the use of the digital pressure gauge in the international comparison of the national standards is expanding. Particularly, in the low-pressure range of $1 \mathrm{kPa}$ or less that normally cannot be generated with a pressure balance, it is common practice to use multiple high-precision digital pressure gauges as the transfer device. Also, in the international comparison that involves transportation in vast areas such as the Asia Pacific, United States, or Africa, there is greater transportation merit in using the small and lightweight digital pressure gauge as the transfer device, rather than the large and heavy pressure balance, as mentioned in subchapter 6.2.

For the remote calibration indicated as an example of incorporating the digital pressure gauge into the Japanese national standard, several similar technological developments have been reported around the world. However, currently, the remote calibration service for pressure gauge is established only at AIST. Several national metrology institutes around the world are interested in this remote calibration technology, and if the issues such as the reduction of calibration cost and the building of the framework for the calibration lab accreditation can be solved, as mentioned in subchapter 7.1, its use is expected to expand overseas as well as in Japan.

\section{Development of remote calibration technology for quantities other than pressure Question (Akira Ono)}

I think the description in this paper is an example where increased performance of the transfer device had a major effect on the entire standards system. Are there any examples for quantities other than pressure?

Also, can you please briefly describe what approaches were taken for quantities other than pressure in the NEDO project for the remote calibration mentioned in subchapter 6.3?

\section{Answer (Tokihiko Kobata)}

Recently there have been various efforts to increase the performance of the transfer device (transfer standard) for various quantities, and the effect of the transfer device on the respective standard system is increasing. For quantities other than pressure, an example where the increased performance of the transfer device had great effect on the standard system is in the temperature measurement as described in Synthesiology [Vol. 3, No. 1, pp. 26-42 (Jul. 2010)]. In that paper, Arai et al. built the standard system for high temperature, and effectively utilized the newly developed thermocouple as the transfer device to maintain the reliability of the measurement.

In NEDO's e-trace Project, the development of the remote calibration technology was done to efficiently provide various measurement standards. The various measurement standards that were studied in the e-trace project can be roughly categorized into two groups. One is the standard for the remote calibration using the international positioning system (GPS) and the optical fiber network, and the other is the standard using the portable transfer device. The former includes the standards for time (frequency), length (wavelength and optical fiber application), and electricity (direct current voltage), while the latter includes the standards for electricity (AC voltage, low frequency impedance), radiation, three-dimensional coordinate measurement, vibration and acceleration, pressure, and temperature. In the time (frequency) standard that is a typical example of the former, the remote calibration technology for frequency was developed using the method mediated by the GPS satellite. For the latter, we developed the technology for conducting the remote calibration by sending the transfer device to the client-designated place and then using the Internet to do the calibration, in the same way as in the pressure standard described in subchapter 6.3. 